\title{
Subitizing in congenitally blind adults
}

\author{
LUDOVIC FERRAND \\ CNRS and University Blaise Pascal, Clermont-Ferrand, France \\ KeVIN J. RIGGS \\ London Metropolitan University, London, England \\ AND \\ Julie Castronovo \\ University of Leeds, Leeds, England \\ and Université Catholique de Louvain, Louvain, Belgium
}

\begin{abstract}
We investigated the role of vision in tactile enumeration within and outside the subitizing range. Congenitally blind and sighted (blindfolded) participants were asked to enumerate quickly and accurately the number of fingers stimulated. Both groups of participants enumerated one to three fingers quickly and accurately but were much slower and less accurate with four to nine fingers. Within the subitizing range, blind participants performed no differently from both sighted (blindfolded) and sighted-seeing participants. Outside of the subitizing range, blind and sighted-seeing participants showed better performance than did sighted-blindfolded participants, suggesting that lack of access to the predominant sensory modality does affect performance. Together, these findings further support the claim that subitizing is a general perceptual mechanism and demonstrate that vision is not necessary for the development of the subitizing mechanism.
\end{abstract}

One of the oldest findings in experimental psychology is the discontinuity in enumeration of visual objects between small numbers of items (one to three) and larger numbers (e.g., Jevons, 1871; Warren, 1897). Enumerating small sets of one to three items is fast and accurate (with a shallow slope of 40-80 msec/item) but suddenly becomes slow and less accurate beyond this range, showing a linear increase of about 200-400 msec/item (e.g., Mandler \& Shebo, 1982; Trick \& Pylyshyn, 1993, 1994; see Trick, 2008, for a review). This discontinuity has traditionally been fitted to a bilinear function (Trick \& Pylyshyn, 1993) and has generally been taken to reflect two separate processes: a preattentive parallel process for small numerosities, called subitizing (a term coined by Kaufman, Lord, Reese, \& Volkmann, 1949), and an attentive serial process for larger ones, referred to as counting/estimating.

Supplementary support for this distinction between enumeration within and outside the subitizing range also has come from brain-imaging studies (Nan, Knösche, \& Luo, 2006; Piazza, Giacomini, Le Bihan, \& Dehaene, 2003; Sathian et al., 1999; Vuokko, Niemivirta, \& Helenius, 2009). For instance, using event-related fMRI, Piazza et al. reported a set of bilateral frontoparietal regions that were intensively active during counting, but to a much lesser extent during subitizing. Those regions showed no increase in activation from numerosity one to three, but a sudden increase between numerosities three and four, and a linear increase from four onward. These studies support a two-process model with distinct neural operations underlying subitizing and counting.

For over 100 years, researchers have focused on $v i$ sual enumeration, and many theories have proposed that subitizing is a process in visual perception (Dehaene \& Cohen, 1994; Mandler \& Shebo, 1982; Olivers \& Watson, 2008; Revkin, Piazza, Izard, Cohen, \& Dehaene, 2008; Trick, 2008; Trick \& Pylyshyn, 1994). Recently, however, Riggs et al. (2006) investigated enumeration in tactile perception and reported a clear discontinuity between one to three and four to six items for both correct naming times and accuracy. Riggs et al.'s results are of theoretical importance because they suggest that subitizing is a general perceptual process, not one restricted to the visual modality. Similar results were recently replicated in active touch by Plaisier, Bergmann Tiest, and Kappers (2009a) and in the auditory domain by Camos and Tillmann (2008).

Taken together, these data suggest that vision is not necessary for the subitizing mechanism to develop or operate. However, there are reasons to think that such a suggestion is premature, and in the research reported here, we further investigated the role of vision in tactile enumeration within and outside the subitizing range. First, there is a concern with Riggs et al.'s (2006) methodology. In that study, participants had their fingertips stimulated by small metal rods and were asked to enumerate the number of fin- 
gers stimulated as quickly and as accurately as possible. However, during the testing phase, the participants could see their hands, so we cannot rule out the possibility that they used visual information to enumerate the number of fingers stimulated. Had they done so, it would undermine the claim that subitizing occurs in tactile perception. In the present study, we therefore tested a group of sighted but blindfolded participants on the original Riggs et al. task.

Second, although the Riggs et al. (2006) data suggest that the subitizing mechanism relies on spatial processing (not necessarily visual), vision has nevertheless been considered important in the acquisition of spatial knowledge (Fraiberg, 1977; Hartlage, 1969; von Senden, 1960; see Gaunet, Martinez, \& Thinus-Blanc, 1997, for a review). Interestingly, the large body of research conducted on this issue has led to discrepant results, with early visual deprivation appearing to entail both increased (e.g., Collignon, Renier, Bruyer, Tranduy, \& Veraart, 2006) and altered (e.g., Gaunet et al., 1997) spatial abilities. For example, Gaunet and Rossetti (2006) provided a good illustration of the different effects of visual deprivation on spatial behavior. They used a pointing task at proximal memorized proprioceptive targets but took different measures (e.g., absolute distance estimation, amplitude and direction of estimates) to assess the spatial representation involved in proprioception. Their results showed that within the same task, blind participants presented different results, according to the different pointing parameters measured (e.g., increased capacity in absolute distance estimation but altered organization for amplitude and direction estimates).

Third, spatial processing has also been linked to numerical cognition, on the basis of the assumption that they share some common underlying mechanisms - notably, in tasks such as enumeration (for reviews, see de Hevia, Vallar, \& Girelli, 2008; Hubbard, Piazza, Pinel, \& Dehaene, 2005). Moreover, researchers have reported that early visual deprivation and the subsequent experience with numerical information enhances participants' numerical estimation abilities outside the subitizing range (Castronovo \& Seron, 2007).

Therefore, to more firmly establish the role of vision in tactile enumeration within and outside the subitizing range, we tested a group of congenitally blind adults. If we were to find evidence of subitizing in these participants, it would (1) rule out the possibility that visual processing was used in the Riggs et al. (2006) study and would consolidate the finding that subitizing is a general perceptual mechanism, (2) be a convincing demonstration that vision is not necessary for the subitizing mechanism to develop or operate, and (3) further our understanding of the role that vision plays in the development of numerical processing.

\section{METHOD}

\section{Participants}

Nine congenitally blind and 9 sighted (blindfolded) people participated in the experiment. Blind participants ( 6 females, 3 males) were blind at birth or had lost sight before the end of their first year (see Table 1). All were recruited through announcements in various French institutions for the blind and were native French speakers and Braille readers. Their mean age was 26 years. None had neurological or motor deficits.

Sighted (blindfolded) participants (6 females, 3 males) were native French-speaking volunteers. Each was chosen to match a blind participant in terms of age, gender, and level of education. Their mean age was 26 years. They were blindfolded during the task.

All the participants were right-handed. They gave written informed consent to inclusion in this study and received payment $(€ 25)$ for their participation.

\section{Apparatus and Stimuli}

The same apparatus as that constructed by Riggs et al. (2006) was used. It consisted of two boxes (381 mm long, $220 \mathrm{~mm}$ wide, $64 \mathrm{~mm}$ high) used to stimulate the fingertips of each hand. Five holes, one for each digit, were drilled into each box to allow small metal rods (6 $\mathrm{mm}$ long, $1.6 \mathrm{~mm}$ in diameter) to protrude from the box and stimulate the fingertips. The fingers did not rest directly on the boxes but rested on small rubber rings $(4 \mathrm{~mm}$ high, $17-\mathrm{mm}$ outer diameter, 7-mm inner diameter) to help keep the fingertips in place during the testing session. The metal rods used to stimulate the fingertips were activated by electromagnets and shot up from the boxes, at a speed of $6.33 \mathrm{~m} / \mathrm{sec}$, to a height $2 \mathrm{~mm}$ above the top of the rubber rings, applying a force to the fingertips of $5 \mathrm{~N}$. The stimulation trials were controlled via a PC running dedicated software written in Delphi. The participants responded into a microphone (Sennheiser e855) attached to a voice-activated relay, which stopped the computer's clock.

\section{Procedure}

Exactly the same procedure as that in Riggs et al. (2006) was used. After a training period of 40 trials, each participant received 50 trials with each of 10 numerosities, for a total of 500 trials. The participants rested for a few minutes after every 100 trials. Fingertips were stimulated until the participants responded by speaking into a microphone attached to a voice key recording naming times to the nearest millisecond and retracting the rods. There was a 5,000-msec interval between the vocal response and the next stimulus presenta-

Table 1

Characteristics of the Blind Participants

\begin{tabular}{clcclc}
\hline $\begin{array}{c}\text { Participant } \\
\text { Number }\end{array}$ & \multicolumn{1}{c}{$\begin{array}{c}\text { Education } \\
\text { Level }\end{array}$} & Gender & Age & \multicolumn{1}{c}{ Etiology } & $\begin{array}{c}\text { Age of Onset } \\
\text { of Blindness }\end{array}$ \\
\hline 1 & University & F & 24 & Glaucoma & 4 months \\
2 & Higher education & M & 20 & Congenital glaucoma & 0 \\
3 & University & F & 25 & Congenital glaucoma & 0 \\
4 & Secondary school & F & 26 & Genetic retinitis pigmentosa & 0 \\
5 & Higher education & F & 27 & Congenital glaucoma & 0 \\
6 & Secondary school & M & 24 & Retinoblastoma & 3 months \\
7 & Higher education & M & 26 & Congenital malformation & 0 \\
8 & Secondary school & F & 30 & Measles (unknown) & 6 months \\
9 & Higher education & F & 31 & Congenital disease & 0 \\
\hline
\end{tabular}


Table 2

Mean Correct Naming Latencies (in Milliseconds) and Standard Deviations As a Function of Group (Congenitally Blind, Sighted Blindfolded, and Sighted Seeing) and Numerosity

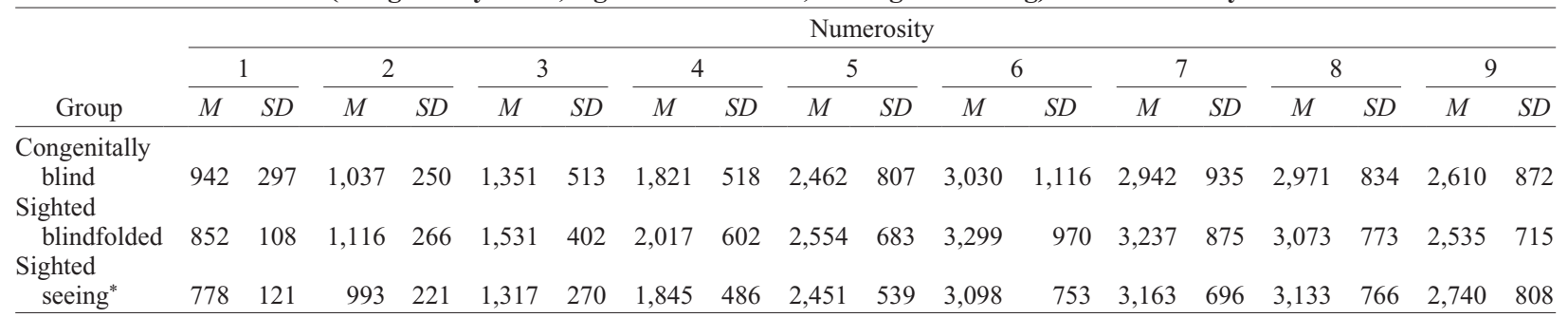

${ }^{*}$ Taken from Riggs et al. (2006).

Table 3

Mean Accuracy and Standard Deviations As a Function of Group

(Congenitally Blind, Sighted Blindfolded, and Sighted Seeing) and Numerosity

\begin{tabular}{|c|c|c|c|c|c|c|c|c|c|c|c|c|c|c|c|c|c|c|}
\hline \multirow[b]{3}{*}{ Group } & \multicolumn{18}{|c|}{ Numerosity } \\
\hline & \multicolumn{2}{|c|}{1} & \multicolumn{2}{|c|}{2} & \multicolumn{2}{|c|}{3} & \multicolumn{2}{|c|}{4} & \multicolumn{2}{|c|}{5} & \multicolumn{2}{|c|}{6} & \multicolumn{2}{|c|}{7} & \multicolumn{2}{|c|}{8} & \multicolumn{2}{|c|}{9} \\
\hline & $M$ & $S D$ & $M$ & $S D$ & $M$ & $S D$ & $M$ & $S D$ & $M$ & $S D$ & $M$ & $S D$ & $M$ & $S D$ & $M$ & $S D$ & $M$ & $S D$ \\
\hline blind & 99.56 & 0.88 & 98.00 & 3.00 & 96.22 & 4.18 & 84.20 & 14.09 & 79.00 & 13.02 & 65.56 & 20.91 & 47.78 & 17.53 & 50.11 & 20.07 & 53.00 & 26.62 \\
\hline \multicolumn{19}{|l|}{ Sighted } \\
\hline blindfolded & 99.11 & 2.03 & 97.33 & 4.00 & 91.56 & 6.77 & 70.56 & 18.47 & 54.67 & 21.05 & 43.22 & 23.08 & 38.00 & 22.32 & 38.89 & 20.75 & 49.00 & 22.47 \\
\hline \multicolumn{19}{|l|}{ Sighted } \\
\hline seeing* & 99.50 & 0.89 & 98.25 & 2.41 & 93.25 & 5.74 & 74.88 & 15.32 & 65.50 & 18.57 & 46.75 & 27.43 & 46.13 & 23.42 & 49.00 & 21.88 & 60.63 & 22.23 \\
\hline
\end{tabular}

*Taken from Riggs et al. (2006).

tion. Stimulus sizes were presented randomly across all 10 fingers. ${ }^{1}$ The distance between the two hands resting on the boxes was $30 \mathrm{~cm}$, with the two arms (and hands) parallel to each other and perpendicular to the body. The experimenter was present in the room to type the participant's numerical response into the computer, and the experiment lasted approximately $1.5 \mathrm{~h}$.

\section{RESULTS}

Since the participants presented ceiling performances for the target magnitude 10, we therefore restricted our analysis to set sizes of one to nine fingers. For the purpose of comparison, we added the results obtained by Riggs et al. (2006) with sighted-seeing participants $(n=16)$. For all the groups (blind, sighted blindfolded, and sighted seeing), we found that both accuracy and correct naming times varied with numerosity (see Tables 2 and 3).

\section{Correct Naming Times}

A $3 \times 9$ (group $\times$ numerosity) ANOVA performed on the correct naming times showed a significant main effect of numerosity $\left[F(8,248)=150.20, p<.001, \eta^{2}=\right.$ .83] but no main effect of group $[F(2,31)=0.107]$ and no group $\times$ numerosity interaction $[F(16,248)=0.556]$. Planned comparisons showed a significant effect of numerosity for blind individuals $[F(8,64)=26.26, p<.001$, $\left.\eta^{2}=.77\right]$, sighted-blindfolded individuals $[F(8,64)=$ 45.71, $p<.001, \eta^{2}=.85$ ], and sighted-seeing individuals $\left[F(8,120)=106.92, p<.001, \eta^{2}=.88\right]$.

The subitizing range was determined with trend analyses (see, e.g., Trick \& Pylyshyn, 1993). Separate ANOVAs were carried out on the response time (RT) data for numerosities $1-3,1-4$, and so on, and the results were scrutinized for the appearance of a significant quadratic component. This divergence from a linear increase in RT suggests the deployment of a new process for the last numerosity added. For all groups, the first appearance of a significant quadratic trend was in the $1-4$ range $(p<.05$ for all). Consequently, the subitizing range was considered to be $1-3$.

We fitted linear and bilinear models to our latency data from all the groups of participants (only for the range 1-6, as is typically done in the literature). We then computed goodness-of-fit statistics comparing data from the models with observed data. For both groups of participants (congenitally blind and sighted blindfolded), data from the linear model differed significantly from those obtained empirically (blind, $\chi^{2}=151.91, p<.001$; sighted blindfolded, $\chi^{2}=75.53, p<.001$ ), but data from the bilinear model (pivoted around array size 3 ) was a good fit for both groups (blind, $\chi^{2}=7.66, p=.18$; sighted blindfolded, $\left.\chi^{2}=6.16, p=.29\right)$. This pattern was also found when we computed goodness of fit on the original Riggs et al. (2006) data (linear, $\chi^{2}=124.29, p<.001$; bilinear, $\left.\chi^{2}=2.07, p=.84\right)$. This fitting exercise confirms that the bilinear function provides a good fit to our data, whereas a linear function does not.

Finally, we fitted linear functions relating naming RT to numerosity within and beyond the subitizing range. For numerosities of $1-3$, the regression equation was $\mathrm{RT}=$ $634+255 \mathrm{~N}(r=.96)$ for blind individuals and RT $=$ $487+339 \mathrm{~N}(r=.99)$ for sighted-blindfolded individuals. For numerosities of $4-6$, the regression equation was $\mathrm{RT}=1,228+605 \mathrm{~N}(r=.99)$ for blind individuals and $\mathrm{RT}=1,341+641 \mathrm{~N}(r=.99)$ for sighted-blindfolded in- 


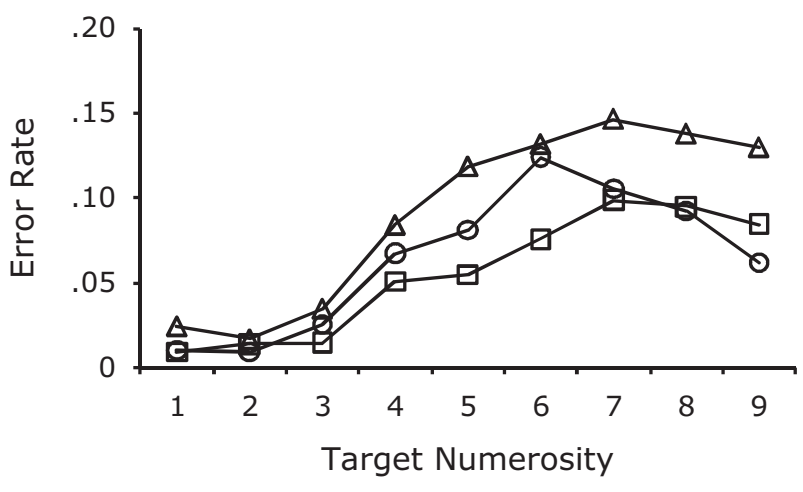

Figure 1. Absolute error rates as a function of the number of fingers stimulated (blind individuals, open squares; sightedblindfolded individuals, open triangles). For comparison, the figure also shows Riggs et al.'s (2006, p. 272, Figure 1) results obtained in tactile perception for sighted-seeing individuals (open circles).

Table 4

Mean |ER| and Standard Deviations As a Function of Group (Congenitally Blind, Sighted Blindfolded, and Sighted Seeing), Within and Outside Subitizing Range

\begin{tabular}{|c|c|c|c|c|}
\hline \multirow[b]{2}{*}{ Group } & \multicolumn{2}{|c|}{$\begin{array}{c}\text { Within } \\
\text { Subitizing }\end{array}$} & \multicolumn{2}{|c|}{$\begin{array}{c}\text { Outside } \\
\text { Subitizing }\end{array}$} \\
\hline & $M$ & $\frac{S D}{S D}$ & $M$ & $S D$ \\
\hline Congenitally blind & .012 & .003 & .077 & .020 \\
\hline Sighted blindfolded & .025 & .009 & .125 & .022 \\
\hline Sighted seeing ${ }^{*}$ & .015 & .090 & .089 & .024 \\
\hline
\end{tabular}

*Taken from Riggs et al. (2006).

dividuals. These results are very similar to those reported by Riggs et al. (2006) for sighted-seeing individuals (for $1-3, \mathrm{RT}=490+270 \mathrm{~N}, r=.99$; for $4-6, \mathrm{RT}=1,212+$ $627 \mathrm{~N}, r=.99)$. The average correct naming time for one to three fingers was significantly quicker than the average naming time for four to six fingers for blind [255 vs. $605 \mathrm{msec} /$ item; $\left.F(1,8)=39.98, p<.001, \eta^{2}=.83\right]$, sighted-blindfolded [339 vs. $641 \mathrm{msec} /$ item; $F(1,8)=$ $\left.77.75, p<.001, \eta^{2}=.90\right]$, and sighted-seeing [270 vs. $627 \mathrm{msec} /$ item; $\left.F(1,15)=181.67, p<.001, \eta^{2}=.92\right]$ individuals. The slopes within and outside the subitizing range did not differ significantly between groups (all $\left.F_{\mathrm{S}}<1\right)$.

\section{Accuracy}

There was a clear discontinuity in accuracy performance, which was nearly perfect for one to three fingers but was severely impaired in the range of four to nine fingers (see Table 3 ). A $3 \times 9$ (group $\times$ numerosity) ANOVA performed on accuracy showed a significant main effect of numerosity $\left[F(8,248)=110.30, p<.001, \eta^{2}=.78\right]$, no main effect of group $[F(2,31)=1.36]$, and a significant group $\times$ numerosity interaction $[F(16,248)=1.85, p<$ $\left..05, \eta^{2}=.11\right]$. The interaction came from the fact that, as in Castronovo and Seron (2007), blind individuals showed less of an increasing tendency to misestimate target magnitudes, as compared with the two other groups, especially outside the subitizing range. Indeed, planned comparisons showed a less significant effect of numerosity for blind individuals $\left[F(8,64)=24.34, p<.001, \eta^{2}=.75\right]$ than for sighted-blindfolded individuals $[F(8,64)=46.44$, $\left.p<.001, \eta^{2}=.85\right]$ and for sighted-seeing individuals $\left[F(8,120)=53.14, p<.001, \eta^{2}=.78\right] .^{2}$

\section{Error Rates}

To investigate how inaccurate the three groups' estimations were within and outside the subitizing range, we conducted one-way ANOVAs on the absolute error rates $[|\mathrm{ER}|=$ (response - target magnitude)/target magnitude $]$, followed by Tukey post hoc tests to compare $|\mathrm{ER}|$ across the groups (see Figure 1 and Table 4). Within the subitizing range, the group effect did not reach significance $[F(2,8)=2.64, p>.15]$, indicating that the three groups of participants presented a similar $|\mathrm{ER}|$. However, outside the subitizing range, the group effect was significant $\left[F(2,17)=7.781, p=.005, \eta^{2}=.48\right]$. Tukey post hoc comparisons indicated that the sighted-blindfolded participants presented a larger $|\mathrm{ER}|$, as compared with the congenitally blind $(p=.005)$ and the sighted-seeing $(p<$ $.05)$ participants, who showed a similar $|\mathrm{ER}|(p>.6)$.

\section{$\log (\mathrm{CV})$}

To further examine the differences between groups within and outside the subitizing range, coefficients of variation $(\mathrm{CV}=$ response standard deviation/mean response) were computed (see Figure 2 and Table 5). Oneway ANOVAs on $\log (\mathrm{CV})$ followed by Tukey post hoc

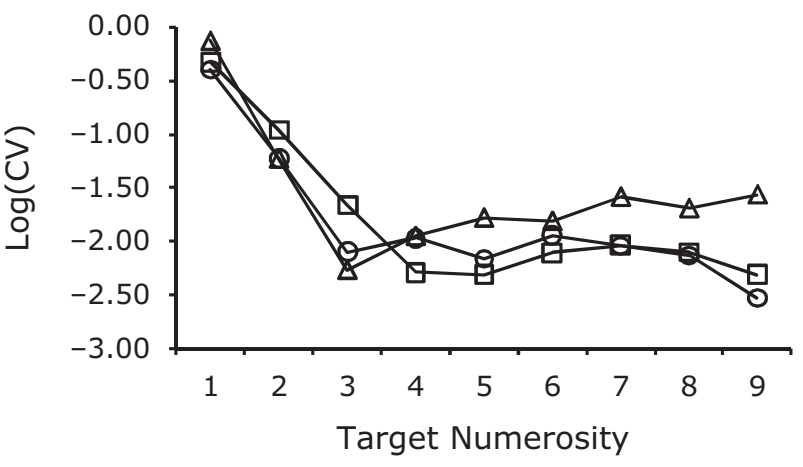

Figure 2. $\log (\mathrm{CV})$ as a function of the number of fingers stimulated (blind individuals, open squares; sighted-blindfolded individuals, open triangles). For comparison, the figure also shows Riggs et al.'s (2006, p. 272, Figure 1) results obtained in tactile perception for sighted-seeing individuals (open circles).

Table 5

Mean $\log (\mathrm{CV})$ and Standard Deviations As a Function of Group (Congenitally Blind, Sighted Blindfolded, and Sighted Seeing), Within and Outside Subitizing Range

\begin{tabular}{lccccc}
\hline & \multicolumn{2}{c}{$\begin{array}{c}\text { Within } \\
\text { Subitizing }\end{array}$} & & \multicolumn{2}{c}{$\begin{array}{c}\text { Outside } \\
\text { Subitizing }\end{array}$} \\
\cline { 2 - 3 } \cline { 5 - 6 } Group & $M$ & $S D$ & & $M$ & $S D$ \\
\hline Congenitally blind & -0.98 & 0.67 & & -2.20 & 0.12 \\
Sighted blindfolded & -1.21 & 1.08 & & -1.74 & 0.15 \\
Sighted seeing* & -1.23 & 0.85 & & -2.13 & 0.21 \\
\hline
\end{tabular}

${ }^{*}$ Taken from Riggs et al. (2006). 
tests showed that within the subitizing range, the three groups of participants presented a similar $\log (\mathrm{CV})$, since the group effect was not significant $[F(2,8)=0.077]$. However, outside the subitizing range, the group effect $\left[F(2,17)=13.69, p<.001, \eta^{2}=.62\right]$ reflected the fact that sighted-blindfolded participants presented a greater $\log (\mathrm{CV})$ than did congenitally blind $(p=.001)$ and sighted-seeing $(p<.005)$ participants, who presented a similar $\log (\mathrm{CV})(p>$.7). This result indicates that sightedblindfolded participants showed greater variability in their estimation outside the subitizing range, as compared with the two other groups of participants.

\section{DISCUSSION}

The aim of this study was to investigate the role of vision in tactile numerical estimation within and outside the subitizing range. A group of congenitally blind participants and a group of sighted-blindfolded participants were asked to enumerate as quickly and as accurately as possible the number of fingers that had been stimulated by small metal rods. The results demonstrate a discontinuity between small numerosities (1-3) and larger numerosities (4-9). This discontinuity in the naming slope was observed for blind participants, sighted-blindfolded participants, and sighted-seeing participants and replicated the standard bilinear function reported in the literature on visual subitizing ${ }^{3}$ (e.g., Logan \& Zbrodoff, 2003; Mandler \& Shebo, 1982; Trick, 2008). Moreover, the three groups of participants presented similarly accurate and fast performance $[|\mathrm{ER}|$ and $\log (\mathrm{CV})]$ within the subitizing range.

Therefore, our data clearly demonstrate that subitizing is a general perceptual mechanism that extends beyond visual to tactile (Plaisier et al., 2009a) and auditory (Camos \& Tillmann, 2008) stimuli. They also allow us to rule out the possibility that participants in the original Riggs et al. (2006) study enumerated the number of fingers stimulated within the subitizing range using visual information, since sighted-blindfolded participants performed similarly to sighted-seeing participants for small array sizes.

For a long time, it has been assumed that following their sensory deficit, blind people develop stronger sensory abilities in their remaining senses as compensatory mechanisms. However, recent studies suggest that blind people might not present increased sensory hearing and touch per se but, rather, might show better top-down cognitive processes, such as attention, when information is accessed in their remaining sensory modalities (Collignon et al., 2006; Lewald, 2002; Röder, Rösler, \& Neville, 1999; Zwiers, Van Opstal, \& Cruysberg, 2001). In view of these findings, what do our results tell us about the subitizing mechanism?

The performance of blind participants in the subitizing range gives further support to the view that subitizing is a low-level preattentive process. The fact that blind participants did not show better subitizing performance, as compared with the other two groups, suggests that they could not rely on greater attentional abilities afforded them through the use of their predominant sensory modality (touch).

Outside of the subitizing range, congenitally blind participants showed performance similar to that of the sighted-seeing participants, although both groups performed better than the sighted-blindfolded group. One possibility is that the congenitally blind participants and the sighted-seeing participants allocated attentional resources equally - maybe because each group was able to rely on their own predominant sensory modality (i.e., vision for the sighted-seeing group and touch for the congenitally blind group). Future work is needed to provide a fuller explanation of these findings, but, at the very least, these data provide further evidence that vision is not necessary to the elaboration of numerical abilities outside of the subitizing range (Castronovo \& Seron, 2007). However, given that sighted-blindfolded participants showed worse performance, as compared with the two other groups, at larger array sizes, our results also show that, even though vision is not necessary per se, the input modality used for enumeration outside the subitizing range is important and can affect participants' performance if they cannot access numerical information through their predominant sense.

In summary, our findings add to the growing body of evidence suggesting that the rapid enumeration of three items takes place through most, if not all, of our senses. Subitizing is a general perceptual mechanism extending to auditory and tactile processing. Importantly, our results demonstrate for the first time that visual processing is not necessary for the development of a rapid enumeration mechanism for small numbers of items. They also provide further evidence that the absence of vision since birth does not preclude the development of estimation processes beyond the subitizing range.

\section{AUTHOR NOTE}

Correspondence concerning this article should be addressed to L. Ferrand, LAPSCO, CNRS and University Blaise Pascal, 34 Avenue Carnot, 63000 Clermont-Ferrand, France (e-mail: ludovic.ferrand@ univ-bpclermont.fr).

Note-Accepted by Cathleen M. Moore's editorial team.

\section{REFERENCES}

Camos, V., \& Tillmann, B. (2008). Discontinuity in the enumeration of sequentially presented auditory and visual stimuli. Cognition, 107, 1135-1143.

Castronovo, J., \& Seron, X. (2007). Numerical estimation in blind subjects: Evidence of the impact of blindness and its following experience. Journal of Experimental Psychology: Human Perception \& Performance, 33, 1089-1106.

Collignon, O., Renier, L., Bruyer, R., Tranduy, D., \& Veraart, C. (2006). Improved selective and divided spatial attention in early blind subjects. Brain Research, 1075, 175-182.

DeHAEne, S., \& CoHen, L. (1994). Dissociable mechanisms of subitizing and counting: Neuropsychological evidence from simultanagnosic patients. Journal of Experimental Psychology: Human Perception \& Performance, 20, 958-975.

de Hevia, M. D., Vallar, G., \& Girelli, L. (2008). Visualizing numbers in the mind's eye: The role of visuo-spatial processes in numerical abilities. Neuroscience \& Biobehavioral Reviews, 32, 1361-1372.

FraiberG, S. (1977). Insights from the blind: Comparative studies of blind and sighted infants. New York: Basic Books. 
Gaunet, F., Martinez, J.-L., \& Thinus-Blanc, C. (1997). Early-blind subjects' spatial representation of manipulatory space: Exploratory strategies and reaction to change. Perception, 26, 345-366.

Gaunet, F., \& Rossetti, Y. (2006). Effects of visual deprivation on space representation: Immediate and delayed pointing toward memorised proprioceptive targets. Perception, 35, 107-124.

Hartlage, L. C. (1969). Verbal tests of spatial conceptualization. Journal of Experimental Psychology, 80, 180-182.

Hubbard, E. M., Piazza, M., Pinel, P., \& Dehaene, S. (2005). Interactions between number and space in parietal cortex. Nature Reviews Neuroscience, 6, 435-448.

JEVONS, W. S. (1871). The power of numerical discrimination. Nature, 3, 281-282.

Kaufman, E. L., Lord, M. W., Reese, T. W., \& Volkmann, J. (1949). The discrimination of visual number. American Journal of Psychology, 62, 498-525.

LEWALD, J. (2002). Opposing effects of head position on sound localization in blind and sighted human subjects. European Journal of Neuroscience, 15, 1219-1224.

Logan, G. D., \& ZBrodoff, N. J. (2003). Subitizing and similarity: Toward a pattern-matching theory of enumeration. Psychonomic Bulletin \& Review, 10, 676-682.

Mandler, G., \& Shebo, B. J. (1982). Subitizing: An analysis of its component processes. Journal of Experimental Psychology: General, $111,1-22$

Nan, Y., KNösche, T. R., \& Luo, Y.-J. (2006). Counting in everyday life: Discrimination and enumeration. Neuropsychologia, 44, 1103-1113.

Olivers, C. N. L., \& Watson, D. G. (2008). Subitizing requires attention. Visual Cognition, 16, 439-462.

Piazza, M., Giacomini, E., Le Bihan, D., \& Dehaene, S. (2003). Single-trial classification of parallel pre-attentive and serial attentive processes using functional magnetic resonance imaging. Proceedings of the Royal Society B, 270, 1237-1245.

Plaisier, M. A., Bergmann Tiest, W. M., \& Kappers, A. M. L. (2009a). One, two, three, many-Subitizing in active touch. Acta Psychologica, 131, 163-170.

Plaisier, M. A., Bergmann Tiest, W. M., \& Kappers, A. M. L. (2009b). Salient features in 3-D haptic shape perception. Attention, Perception, \& Psychophysics, 71, 421-430.

Revkin, S. K., Piazza, M., Izard, V., Cohen, L., \& Dehaene, S. (2008). Does subitizing reflect numerical estimation? Psychological Science, 19, 607-614.

Riggs, K. J., Ferrand, L., Lancelin, D., Fryziel, L., Dumur, G., \& Simpson, A. (2006). Subitizing in tactile perception. Psychological Science, 17, 271-272.

RöDER, B., RöSLER, F., \& Neville, H. J. (1999). Effects of interstimulus interval on auditory event-related potentials in congenitally blind and normally sighted humans. Neuroscience Letters, 264, 53-56.

Sathian, K., Simon, T. J., Peterson, S., Patel, G. A., Hoffman, J. M., \& Grafton, S. T. (1999). Neural evidence linking visual object enumeration and attention. Journal of Cognitive Neuroscience, 11, 36-51.
TRICK, L. M. (2008). More than superstition: Differential effects of featural heterogeneity and change on subitizing and counting. Perception \& Psychophysics, 70, 743-760.

Trick, L. M., \& Pylyshyn, Z. W. (1993). What enumeration studies can show us about spatial attention: Evidence for limited capacity preattentive processing. Journal of Experimental Psychology: Human Perception \& Performance, 19, 331-351.

TRICK, L. M., \& PYLYShyn, Z. W. (1994). Why are small and large numbers enumerated differently? A limited-capacity preattentive stage in vision. Psychological Review, 101, 80-102.

von SENDEN, M. (1960). Space and sight: The perception of space and shape in the congenitally blind before and after operation (P. Heath, Trans.). Glencoe, IL: Free Press.

Vuokko, E., Niemivirta, M., \& Helenius, P. (2009, December). Different brain mechanisms for subitizing and counting revealed by $M E G$. Poster presented at the Workshop on Learning, Mind, and Brain, Helsinki, Finland.

Warren, H. C. (1897). The reaction time of counting. Psychological Review, 4, 569-591.

Zwiers, M. P., Van Opstal, A. J., \& Cruysberg, J. R. M. (2001). A spatial hearing deficit in early-blind humans. Journal of Neuroscience, 21(9, Art. RC142).

\section{NOTES}

1. The fingers and hands were selected randomly from among 1,023 possible combinations.

2. No further analyses were performed on accuracy, because most of the studies in the subitizing literature have focused on the RT variable, rather than on accuracy. Furthermore, participants could, in principle, respond $100 \%$ correctly at all array sizes and still produce a bilinear function for the latency data. In fact, in the Riggs et al. (2006) study, 2 participants had near-ceiling accuracy ( $96 \%$ and $97 \%)$, but both exhibited a bilinear function for latency data.

3. One reviewer (Derrick Watson) underlined the fact that tactile subitizing RT slopes are much greater here ( $250-300 \mathrm{msec} / \mathrm{item})$ than in visual enumeration studies (typically, around $40 \mathrm{msec} / \mathrm{item}$ and, generally, $<100 \mathrm{msec} /$ item), implying that tactile representations might be noisier than visual representations, leading to slower tactile enumeration rates than visual enumeration rates. Although the tactile subitizing RT slopes are larger than those found in visual studies, they are in agreement with values one would expect from the range of RT slopes found in tactile research (see, in particular, Plaisier, Bergmann Tiest, \& Kappers, 2009b). Thus, these RT differences do not rule out the possibility that similar mechanisms underlie numerosity judgments in both modalities, if we assume that enumeration processes are less efficient in tactile perception than in vision.

(Manuscript received March 25, 2010; revision accepted for publication July 28, 2010.) 